The University of Maine

DigitalCommons@UMaine

Publications

Senator George J. Mitchell Center for Sustainability

Solutions

$4-2013$

\title{
Paradigmatic differences, power, and status: a qualitative investigation of faculty in one interdisciplinary research collaboration in sustainability science
}

Susan K. Gardner

University of Maine, susan.k.gardner@maine.edu

Follow this and additional works at: https://digitalcommons.library.umaine.edu/ mitchellcenter_pubs

\section{Repository Citation}

Gardner, Susan K., "Paradigmatic differences, power, and status: a qualitative investigation of faculty in one interdisciplinary research collaboration in sustainability science" (2013). Publications. 57.

https://digitalcommons.library.umaine.edu/mitchellcenter_pubs/57 
Paradigmatic differences, power, and status: a qualitative investigation of faculty in one interdisciplinary research collaboration on sustainability science

Susan K. Gardner

S. K. Gardner

College of Education and Human Development,

University of Maine, Orono, ME, USA

e-mail: susan.k.gardner@maine.edu 
ABSTRACT

Interdisciplinary research collaborations are fraught with challenges, including the need to overcome paradigmatic differences. The current study sought to understand how 25 faculty members involved in an interdisciplinary endeavor related to sustainability experienced these differences. Using Biglan's classification and Becher and Trowler's conceptualizations, the study found issues related to disciplinary status and hierarchy between the soft and hard scientists involved in the project.

Keywords: Interdisciplinary, Paradigms, Status, Sustainability science

"What interdisciplinary research projects are undertaken, which disciplines are involved, how conflicts are resolved, and the acceptance of the research by the rest of the scientific community are due, in part, to the differentially perceived power of the research and researchers" (MacMynowski 2007, p. 25) 


\section{INTRODUCTION}

Interdisciplinary research has emerged as a major focus of federal funding (e.g., National Science Foundation 2007, 2009; The National Academies 2004) and, consequently, higher education institutions have seen its burgeoning popularity over the past few decades (Klein 2010). Despite its growth, interdisciplinary research is often difficult to carry out, given the inherent challenges of bringing together multiple disciplines and, therefore, multiple ways of knowing and conducting research (Biglan 1973b; Holley 2009; Klein 1990; Lattuca 2001; MacMynowski 2007; Rossini et al. 1979). In particular, disciplines have their own specific cultures, languages, and standards for adequacy (Becher 1981; Clark 1987), as well as their own paradigmatic assumptions (Biglan 1973b).

Described in many different ways (Kuhn 1962), a paradigm can be understood as "a basic set of beliefs that guides action" (Guba 1990). Much of an individual's understanding about research and the way to go about conducting it is generally a product of one's disciplinary culture (Becher and Trowler 2001; Biglan 1973b; Toma 1997), particularly as the discipline will structure those questions that are legitimate and how to go about addressing such questions (Becher 1989; Frost and Jean 2003; Kuhn 1962).

These differences in paradigmatic orientation also have implications for the nature of the collaboration among disciplines, particularly when the disciplines involved do not share paradigmatic assumptions. For example, many interdisciplinary research 
collaborations are often those that remain within one paradigmatic perspective (Creamer 2003). In fact, the "hard" and "soft" terminology that is often used to describe differences in biophysical sciences versus the social sciences, for example, actually refers to the level of paradigmatic consensus among the individuals involved in that discipline, with those in the hard disciplines having more paradigmatic consensus than those in the soft disciplines (Biglan 1973b). In relation to these differences, Biglan commented, "The common framework of content and method which (a paradigm) provides for the members of the field means that their attempts to work together will not be hindered by differences in orientation" (р. 210). In other words, it may be relatively easier for a scientist in a hard discipline to collaborate with a scientist in another hard discipline (i.e., a biologist with a chemist) rather than a hard scientist with a soft scientist (i.e., a biologist with a philosopher).

These differences in paradigmatic assumptions and their resulting terminology have come to represent not only the differences among the disciplines but also to represent status among the disciplines. The terminology such as "hard" and "soft" have taken on a pejorative perspective in popular culture, implying the level of rigor involved in these disciplines, rather than paradigmatic consensus (Berliner 2002; Storer 1967). In turn, researchers in the soft sciences are often faced with negative perceptions of their work when compared to those in the hard sciences (Hedges 1987). Given the need for the crossing of such paradigmatic and, consequently, disciplinary boundaries to solve the world's pressing problems (Jerneck et al. 2011; National Science Foundation 2009; The 
National Academies 2004), a deeper understanding of the issues related to such paradigmatic status and disciplinary hierarchy is needed, particularly among interdisciplinary collaborations involving the biophysical and social sciences, as these often represent the full range of the hard-soft spectrum. MacMynowski (2007) summarized, "Biophysical and social scientists are not just bringing information and different understandings of biophysical and social systems with them. Those knowledge claims have differential power associated with them: within the sciences, between social and biophysical sciences, and between science and society" (p. 23).

And, while a growing body of literature has focused on interdisciplinarity, interdisciplinary collaboration, and interdisciplinary research, relatively little has been empirical in nature. Specifically, while paradigmatic differences are often highlighted as a common obstacle to interdisciplinarity (e.g., Lattuca 2001; Miller et al. 2008; Pallas 2001), relatively little research has occurred that examines these paradigmatic differences and their resulting power dynamics among faculty engaged in such interdisciplinary collaborations, particularly in the context of sustainability science, an inherently interdisciplinary endeavor (Clark et al. 1999; Kates et al. 2000). Moreover, much of what is known about interdisciplinary research has generally been retrospective (Amey and Brown 2004; Lattuca 2001) or anecdotal in nature, thereby, not allowing for a more in-depth understanding of the process of interdisciplinarity as it occurs through empirical study.

The current study sought to understand the paradigmatic differences and resulting 
disciplinary status dynamics of 25 faculty members involved in a large, interdisciplinary research project focused on sustainability science at one research institution in the United States, and how these differences influenced how they saw the interdisciplinary effort in the first year of the project. I begin by highlighting the relevant literature related to interdisciplinary endeavors and disciplinary status in these collaborations, followed by a review of the methods in the study design. I conclude with an overview of the findings, as well as implications for policy, practice, and future research.

Interdisciplinary and disciplinary status

While few would dispute the many potential benefits of interdisciplinary research, the literature is rife with the challenges inherent in conducting it. Derry and Schunn (2005) explained, "Although there is much promise in interdisciplinarity, much need for interdisciplinarity, there are also many dangers" (p. xiv). For many, interdisciplinarity has become synonymous with the concept of "teamwork" (Borrego and Newswander 2010; Klein 2005), leading many to wonder why such work is so inherently difficult and frequently unsuccessful.

An additional confounding element of work that crosses disciplinary lines is often in understanding its definition and boundaries. For example, interdisciplinary research has been defined as:

A mode of research by teams or individuals that integrates information, data, 
techniques, tools, perspectives, concepts, and/or theories from two or more disciplines or bodies of specialized knowledge to advance fundamental understanding or to solve problems whose solutions are beyond the scope of a single discipline or area of research practice. (National Science Foundation 2009)

However, other terms such as integrative research (Stock and Burton 2011), multidisciplinary research, or even transdisciplinary research have also been used to describe research that crosses traditional disciplinary boundaries. For example, interdisciplinarity is distinct from cross-disciplinarity, in which researchers "borrow" methods or understandings from various disciplines and apply them to their own disciplinary studies; or multidisciplinarity, in which scholars will come together to investigate a common problem but will utilize their own disciplinary lenses to do so, later returning to discuss their findings as a group (Holley 2009). Transdisciplinarity, on the other hand, "is the ultimate coordination among the disciplines" (Lattuca2001, p. 116), utilizing frameworks that transcend traditional disciplinary boundaries, such as systems theory (Klein 1990). From another perspective, it is, perhaps, the intended outcome or applicability of the knowledge gained from the disciplinary boundary-crossing effort that ultimately defines its scope: integrative research may include the stakeholders or policymakers (van Kerkhoff 2005) and transdisciplinary research goes beyond mere collaboration of academic participants to include these stakeholders in participatory approaches to create new ways of thinking (Stock and Burton 2011). As such, many have asserted that transdisciplinary research is rarely, if ever, accomplished (Klein 1990; Stock 
and Burton 2011). In this study, I utilize the basic definition as provided by Repko (2008), defining interdisciplinarity as:

The process of answering a question, solving a problem, or addressing a topic that is too broad or complex to be dealt with adequately by a single discipline and draws on disciplinary perspectives and integrates their insights to produce a more comprehensive understanding or cognitive advancement. (p. 12)

Beyond definitions, interdisciplinary research is also challenging from the perspective of the individuals participating in it. For example, scholars have discussed certain traits and qualities being more facilitative of interdisciplinary researchers, such as humility, openmindedness, and a high tolerance of ambiguity (Klein 1990; Repko 2008). This need for open-mindedness has been documented in the research on interdisciplinary team development, wherein Amey and Brown (2004) found individuals in early stages of their projects seeing only their own disciplinary perspectives and becoming more open to other perspectives as the research developed.

From an organizational perspective, the challenges facing interdisciplinary collaboration are voluminous in the literature, including issues related to existing organizational and reward structures, disciplinary socialization, and resulting impediments to communication across disciplinary cultures (e.g., Amey and Brown 2004; Golde and Gallagher 1999; Holley 
2009; Klein 2005, 2010; Lattuca 2001; Repko 2008). In fact, these disciplinary differences often become the sticking point for many interdisciplinary endeavors, particularly as they relate to the status and power differentials apparent among different disciplines. Klein (2005) remarked, "Status is a particularly tenacious problem. Interdisciplinary teams are status systems that reflect external hierarchies and disciplinary chauvinism" (p. 32). And, while disciplinary hierarchy and status can be apparent even within the same fields, as one might see in applied versus basic sciences within the same discipline (Becher and Trowler 2001), the hierarchy is even more salient between the "hard" and "soft" science divide (Klein 2005; MacMynowski 2007).

Biglan (1973b) asserted that "hard" disciplines are those fields in which more paradigmatic consensus exists in regard to research and teaching, whereas the "soft" disciplines exhibit less consensus (see Table 1). These paradigmatic lines are challenging to cross. Connecting his work to interdisciplinarity, Biglan stated, "The common framework of content and method which it provides for the members of the field means that their attempts to work together will not be hindered by differences in orientation," whereas scholars in nonparadigmatic (or soft) fields "must work out a common definition of problems and method of approach before they can begin to work together" (p. 210). Given these fundamental differences in perspective, it is, perhaps, not surprising, then, that the majority of interdisciplinary endeavors may not cross these paradigmatic divides (Creamer 2003).

Specifically, a paradigm includes three main concepts: (a) ontology, or the nature of 
reality (i.e., What exists?); (b) epistemology, or the nature of the relationship between the knower and the known (i.e., How do I know what I know?); and (c) methodology, or the tools or strategies one uses to discover knowledge (i.e., How do I ascertain new knowledge?) (Guba 1990; Kuhn 1962). Guba pointed out that these three concepts "are the starting points or givens that determine what inquiry is and how it is to be practiced" ( $p$. 18).

And, while interdisciplinary efforts can create opportunities for faculty members to gain respect for other disciplines (Frost and Jean 2003) and their respective paradigmatic stances, a danger still exists for partisanship in these endeavors. Miller et al. (2008) explained, "Most interdisciplinary research ends up entitling a single discipline or epistemology, incorporating others in a support or service role-we can refer to this as 'epistemological sovereignty"' (p. 46). Indeed, MacMynowski (2007) discussed how many such interdisciplinary endeavors that cross the hard and soft divide may often group together social sciences in a service role to a biophysical science. At the same time, interdisciplinary endeavors that cross such paradigmatic lines are necessary to solve the complex problems that face the world (National Science Foundation 2009).

A conceptualization of paradigms

Given the fundamental differences inherent in disciplinary paradigms, such an understanding is vital when one considers how interdisciplinary research collaborations 
occur across these boundaries. While it is certainly beyond the scope of this study to provide a thorough discussion of paradigmatic differences and conceptualizations of these paradigms (see also Guba and Lincoln 1994; Kuhn 1962), I present here the widely cited conceptualization of Guba (1990). Guba's presentation of paradigms is one that represents a variety of perspectives used in social science research (i.e., "soft" disciplines), including: (a) positivism, (b) post-positivism, (c) critical theory, and (d) constructivism. Given the larger range of paradigms and epistemologies represented in the soft sciences versus the hard sciences, as well as the lesser ranking of social sciences in these interdisciplinary endeavors, a social science perspective may be the more appropriate way to frame the diversity of approaches available. For example, in the majority of the hard sciences, one may rarely see more than positivist, or less often, post-positivist perspectives in traditional research endeavors, as these disciplines often focus on an experimental method in their scholarly pursuits (Schwandt 1990). In the soft sciences, there is much less consensus on a chosen methodology, thus, owing to the "soft" demarcation (Biglan 1973a). These paradigmatic differences, however, account for many of the roadblocks in interdisciplinary collaborations between biophysical and social sciences (Jerneck et al. 2011; MacMynowski 2007). Below, each of Guba's four paradigms is defined in turn, in relation to their ontological, epistemological, and methodological stances (see Table 2).

\section{Positivism}

Positivism can be described as the "received view that has dominated the formal 
discourse in the physical and social sciences for some 400 years" (Guba and Lincoln 1994, p. 108). Positivists tend to view reality as one that is objective and measurable. The positivist's epistemological assumptions allow for the investigator to determine "how things really are" and "how things really work" (p. 111). Therefore, positivists will tend to utilize methodologies that are experimental/manipulative in nature in order to verify hypotheses. Often, positivists may look for cause-effect in their inquiries, as well as prediction and control.

\section{Post-positivism}

Guba and Lincoln (1994) described post-positivism as a paradigm that has emerged in the past few decades to respond "in a limited way to the most problematic criticisms of positivism" (p. 109). In essence, post-positivists may view reality as through a critical realism stance, wherein reality is still believed to exist but can only be imperfectly understood. Post-positivists may approach inquiry through a much less dualistic view than positivists but will, nevertheless, still adhere to objectivity as an ideal. Methods used by post-positivists may be more naturalistic in scope, in which researchers may collect more situational information. In this way, post-positivists attempt to falsify hypotheses, rather than verifying them.

\section{Critical theory}


Critical theory is "a blanket term denoting a set of several alternative paradigms, including additionally (but not limited to) neo-Marxism, feminism, materialism, and participatory inquiry" (Guba and Lincoln 1994, p. 109). Critical theorists may tend to believe that reality is shaped by social, political, cultural, economic, ethnic, and gender values that may be inappropriately viewed as "real," upon which they may provide their critique. Their epistemological stance is one that imbues a connection between the investigator and the investigated, therefore seeing any findings of inquiry as intrinsically value-mediated. Critical theorists utilize methodologies that are dialogic and transformative in nature. These methods seek to "eliminate false consciousness and energize and facilitate transformation" (Guba 1990, p. 25).

\section{Constructivism}

Finally, constructivism is an "alternative paradigm whose break-away assumption is the move from ontological realism to ontological relativism" (Guba and Lincoln 1994, p. 109). Guba (1990) described constructivism as a reaction to positivism and postpositivism, wherein those paradigms are considered by constructivists to be "badly flawed and must be entirely replaced" (p. 25). From an ontological perspective, constructivists embrace relativism, wherein many interpretations can be made in any inquiry, thereby no absolute truth or falseness can exist. Epistemologically, constructivists choose subjectivity over a false sense of objectivity, especially given the focus on multiple realities. Therefore, the constructivists' methodologies are those that allow them to identify the 
variety of constructions that exist-through hermeneutics and dialectics. Hermeneutics allow for a depiction of "individual constructions as accurately as possible," while the dialectic aspect allows for "comparing and contrasting these individual constructions" ( $p$. 26).

Summary

Taken together, the concepts of paradigmatic assumptions in inquiry and disciplinary hierarchy create a deeper understanding of some of the challenges inherent in interdisciplinary research collaborations that cross both of these boundaries. Guba and Lincoln (1994) remarked, "Differences in paradigm assumptions cannot be dismissed as mere 'philosophical' differences; implicitly or explicitly, these positions have important consequences for the practical conduct of inquiry, as well as for the interpretation of findings and policy choices" (p. 112).

Indeed, the literature on interdisciplinary research has generally discussed the inherent challenges of crossing epistemological and paradigmatic boundaries in these collaborations (Becher 1981; Biglan 1973b; Holley 2009; Jerneck et al. 2011; Lattuca 2001). And, while these challenges are underscored in the literature, relatively little has been empirical in nature. Moreover, given the growing emphasis on interdisciplinary collaborations among biophysical and social sciences (Jerneck et al. 2011; MacMynowski 2007), an empirical examination of the disciplinary status and hierarchy at play in these 
collaborations is warranted in order to create more efficacious collaborations in the future.

METHODS

The current study asked, "How do faculty members engaged in one large interdisciplinary project experience disciplinary status and the paradigmatic boundarycrossing effort?" As such, the study was best suited as a qualitative case study in that it was a "detailed examination of one setting" (Bogdan and Biklen 2003), or a mid-sized, research university in which the interdisciplinary project was situated. Specifically, the project under examination is a $\$ 20$ million, 5-year, federally funded project focused on ecological sustainability and applying research to solve problems in this arena. At the time of the study, in the winter of 2009-2010, the endeavor had only recently received funding and work was beginning on the project that spanned over 25 distinct disciplines, including those from the biophysical sciences, social sciences, humanities, and professional fields. Sub-projects had just begun work in the month that the study commenced, through an internal mini-grant competition reviewed by a representative group of faculty volunteers from the project. In order to be eligible for funding, these subprojects had to demonstrate interdisciplinarity, or the representation and integration of multiple disciplines with an applied focus. As a result, the sub-project proposals that included more disciplines often received relatively more funding than those with fewer disciplines. In this way, the project under investigation became a fascinating case study as 
it, by design, spanned the multiple paradigms of these different disciplinary groups, which is an inherently more challenging form of interdisciplinary collaboration (Creamer 2003).

The Sustainability Project (SP) was an emergent collaborative process, led through shared governance from both social science and biophysical science faculty on one campus. The leadership team of the project, a representative body from the original 33 principal investigators listed on the proposal, was approached and asked to identify individual faculty members who had been integrally involved in the project's success to that point. From the list of 42 faculty affiliated with the SP at the time of the study, 29 faculty members were identified. These 29 individuals were contacted via e-mail to seek their participation, with 25 finally agreeing to be interviewed. The 25 faculty members included faculty from all ranks and diverse disciplines. The faculty interviewed had been at the institution for an average of 9.82 years. The majority of the faculty stated that they had all been involved in what they identified as interdisciplinary endeavors prior to joining the SP, but, upon further elaboration, discussed that these endeavors functioned with disciplines sharing similar paradigmatic stances. Table 3 presents the general disciplines included in the study in accordance with Biglan's $(1973 a, b)$ classification, as well as a balance between the biophysical and social sciences involved in the project.

Qualitative methods were chosen for this study as they allowed for an understanding of the meaning, for the participants in the study, of the events, situations, and actions they are involved with and the accounts they give of their experiences; the particular 
context within which the participants act and the influence the context has on their actions; and the process by which events and actions take place (Maxwell 1996).

After obtaining consent, interviews with the 25 individuals took place in face-to-face settings. Guided by a semi-structured protocol seeking faculty input about the early stages of the project and their collaborations, the interviews lasted 30-120 min, were audiotaped, and then transcribed verbatim. In addition to the interviews, the participants were asked to self-identify their own ontological, epistemological, and methodological positionality in regard to Guba's (1990) classification, due its diversity in paradigmatic, ontological, and methodological stances. Specifically, faculty participants were given a copy of Table 2, with the top line of the table (i.e., positivism, post-positivism, critical theory, constructivism) removed, and were asked to check the columns in which they felt their research stance was best represented. This approach to defining paradigms is similar to that of Toma (1997), but with the added dimension of allowing participants to interpret their own paradigmatic stances instead of assigning my impression of them through analysis. The resulting tables from participants were analyzed and grouped by disciplinary and paradigmatic assumptions as described by Biglan (1973a).

Transcripts were analyzed using the constant comparative method (Glaser 1978) utilizing NVivo software and guided by the frameworks provided by Biglan (1973a), Guba (1990), and Dressel and Marcus (1982) in relation to paradigmatic and disciplinary structures. In particular, this study utilized Glaser's (1978) steps in data analysis, wherein 
first open-coding was utilized to understand the larger dynamics at work in the faculty perceptions of their disciplinary and paradigmatic differences, resulting in a set of themes. Then, further coding was utilized to make explicit the connections between the themes that emerged and corresponded with the existing literature on interdisciplinarity and disciplinary paradigms (Becher 1981; Biglan 1973a, b; Dressel and Marcus 1982; Guba 1990; Guba and Lincoln 1994; Holley 2009). Finally, a third round of coding allowed for a search of concepts that tied into the emic themes (Strauss and Corbin 1998) that emerged from the participants' interviews. Trustworthiness of the data collected and its subsequent analysis was obtained through member checking of participants as well as through peer debriefing, in which a colleague was asked to code a number of transcripts to verify analysis.

\section{FINDINGS}

From the interviews with the 25 faculty members involved in the SP, several areas of emphasis emerged related to their disciplinary perspectives and paradigmatic approaches that they brought to the interdisciplinary endeavor. First, I begin by discussing the paradigmatic assumptions represented among the SP faculty. Second, I discuss the qualitative themes that emerged, including issues related to status and hierarchy.

Paradigmatic assumptions represented 
Once given a copy of Guba's (1990) paradigm table, the 25 faculty members placed themselves in the corresponding categories in relation to how they viewed their ontological, epistemological, and methodological assumptions in their given discipline and their approach to research. As represented in Table 4, the faculty members placed themselves in diverse categories according to the Biglan (1973a) classification. While all faculty were drawn to the SP because of its applied focus through sustainability science research, not all of the disciplines represented necessarily train their researchers with this applied stance. Perhaps not surprisingly, all the faculty members from the hard disciplines $(N=13)$ self-identified as belonging to either the positivist or post-positivist paradigm. When broken down by applied versus pure disciplines, more of the faculty in the hardpure fields identified as belonging to the positivist paradigm $(N=5)$ than those in the hard-applied fields, who were more likely to lean toward the post-positivist paradigm ( $N=$ 3). While the majority of the faculty in the soft disciplines self-identified as belonging to the critical theory and constructivist paradigms or somewhere approaching them $(N=7)$, there were a number of individuals, particularly those from the soft-applied fields such as economics, policy, and business, who were more apt to self-identify as what Guba described as post-positivist $(N=4)$. Soft-pure faculty, such as those in anthropology and communication, were more likely to identify themselves as being in line with critical theorists and constructivists as opposed to post-positivists. Additionally, as seen in Table 4, many of the faculty were more comfortable placing themselves in the interstices between the paradigmatic columns of the table rather than choosing solely one column, echoing the work of Aram (2004). 
Beyond the descriptive data provided through the paradigmatic self-identification, the actual process in itself was fascinating to note. For example, there were three faculty members from the hard disciplines, who, when asked to self-identify, stated frankly, "I don't know what this means" or "I have no idea." Similarly, five other faculty members from hard disciplines required an extensive explanation of what was meant by the terminology presented in Table 3. Several of these faculty members remarked that they found such a process fascinating, with a few individuals asking questions like, "You mean people do research in these other columns? What does that look like?" Faculty members from the soft disciplines, however, were readily able to discuss their own stances and were interested in discussing these in relation to their other team members. While some of these responses could certainly be explained by the hard scientists' unfamiliarity with the social science terminology in the table, it also points to the lack of understanding they had of social or soft sciences as they entered into these interdisciplinary endeavors. The soft scientists, on the other hand, were more than aware of all methods, ontologies, and paradigms, even if they did not conduct research within those frames.

Status and hierarchy

Given the paradigmatic differences present among the 25 faculty members involved in the SP, it was perhaps not surprising that issues related to disciplinary status and hierarchy also emerged. One of the most intriguing themes that emerged from the interviews with 
the SP faculty surrounded the disciplinary divides that the team members were observing. Specifically, faculty discussed seeing two different "camps" of the SP: the physical sciences and the social sciences. In Biglan's (1973a, b) classification, these two camps could also be seen as those in the hard sciences versus those in the soft sciences. A social psychologist on the SP shared, "One of the things that has been a surprise to me is the language people use here, where they keep talking about the differences between the bio-sciences (or whatever the term is that they use), and the social sciences."

Faculty also discussed their own disciplines' place in the larger SP project and how their methodological and epistemological stances played a role in this placement. Specifically, faculty were asked how they felt their disciplines contributed to the larger work of the SP. What emerged from this question, as well as others, was that the faculty members in the soft disciplines were more apt to answer with less confidence and instead with nervous laughter using phrases like, "I’m not sure," and "I have no idea." Conversely, faculty members in the hard disciplines were quick to respond by saying things like, "(My discipline's contribution) is critical," as one hard-pure scientist remarked. A faculty member in a soft-pure discipline explained, "I think I'm easily dismissible (to the hard scientists) because I don't really have a strong claim on objectivity, simply because of the various distances we have between the production of knowledge and, you know, the contours of what counts or not (as knowledge)," whereas a hard-pure scientist said, "I have some difficulty seeing some of the value of some of the social sciences. I have sat through a number of lectures that some of the social science people have given and walked away 
wondering what the point was." Indeed, this feeling of disconnectedness came up in several interviews across paradigms, with several of the hard scientists expressing concern that a lack of respect across these boundaries was an issue to be tackled as the SP progressed. Another hard-pure scientist explained:

At the risk of being too forthright, I think that a lot of the biophysical types feel that the social science types are pretty squishy. One problem is the language problem: "Where were the hypotheses? How much data were gathered? Where are the statistics?" So, there is danger in people not giving due respect to people from other disciplines or people outside their field.

Therefore, almost universally, the faculty who did not identify as hard-pure scientists expressed concern about their role in the project, with some even expressing feelings of marginality within the larger effort. A scientist in the soft-pure disciplines expressed:

I think primarily I feel like the majority of the SP work is going to be done by the biophysical scientists, honestly. I feel like I am in more of a supportive role. And I do feel like a little bit of an outsider from the core team.

Even an engineer in the hard-applied disciplines shared, "As someone who has been part of the scientific disciplines, I feel a little bit excluded from the major focus of the SP, even though it's based on science. How do I fit into the big mission?"

Building the paradigmatic bridge 
While the literature on interdisciplinarity is riddled with the challenges facing such collaborations, the members of the SP in their first year of the project pointed to hope in overcoming these challenges. Many discussed it as a process of overcoming one's ego, while others discussed it as a developmental awakening. Interestingly, it was more often the social scientists on the project that discussed this need to build bridges rather than the biophysical scientists, however.

A soft-pure faculty member explained that, to be able to do this kind of work:

You have to have a high degree of humility. You have to be able to step back and say my way is not the only way or even the most important or even the best or even a good way of thinking about this, which is like the paradigmatic opposite of how we're trained as academics. We're trained to be big heads.

Whereas another soft-pure faculty member expressed, "I think that we are all smart and we all think that we know the way to approach things and that is a dangerous combination. I think that a lot of those in the SP are used to being the big fish in a little pond and now we're all big fish in a little pond and it can be tough."

At the same time, however, another soft-pure faculty member explained how the soft- 
hard divide operates in this process of moving toward humility: "Actually, in some ways, the social scientists have to have an even higher dose of humility in this, especially people like myself, and say I'm willing to work a little harder a little extra to make this work." To engage in humility, one social scientist discussed her process of coming to understand her own disciplinary biases and assumptions in this way:

I did not realize that my first judgment about an encounter with another person from a different discipline was sort of like this dismissal or judgment. Like, "Well, that's just a silly way of looking at things, or that's not right, or that person doesn't quite get it." And that was sort of a dismissive type of judgment that I made. But later, I realized or found out the hard way that really was a defensive reaction, a knee-jerk reaction on my part because this person makes assumptions very differently than I do and has a whole different way of looking at the world. So, I think that my experience there caught me up short because I try to be open-minded and, you know, I try not to have blind spots like that, but it's a big blind spot. And, so, I think if that's happening to me, maybe it happens to other people. So there's another risk of interdisciplinary research: people not seeing their own things and, therefore, sort of getting in turf fights and a lack of, sort of meeting of the minds because of not seeing what you don't see.

\section{DISCUSSION}


The 25 faculty members involved in this large-scale interdisciplinary project discussed many overarching issues relating to their paradigmatic differences and the resulting disciplinary status and hierarchy involved in the SP. These issues are illustrative for other research institutions seeking to encourage such interdisciplinary endeavors. Moreover, the obstacles involved in such collaborations, such as those illustrated in this study, beg for further research, as well as policies and practices to facilitate future endeavors. I discuss the findings from this study within these parameters below.

In this study, the 25 individual faculty members represented almost as many distinct disciplines and nearly all of Biglan's (1973a, b) categories of classification. In turn, the paradigmatic differences among these disciplines already began to create fascinating challenges for the faculty involved in the project, even in its first year. One of the most discussed issues by the SP faculty members was that of the chasm between the hard and soft disciplines. While nearly all the faculty discussed having interdisciplinary experiences in their past, the majority of these collaborations were those that occurred within the same paradigmatic classification. As a result, the challenges that the faculty found in crossing paradigmatic classifications were nontrivial in nature; in fact, interdisciplinary collaborations that cross these paradigmatic boundaries are inherently more challenging (Creamer 2003). The challenges discussed by the faculty included language barriers as well as a deeper understanding of others' methodologies and epistemologies (Guba 1990), as seen in their facility or inability to discuss paradigmatic frames. Given the fact that many of these hard scientists were already working with the soft scientists in their sub-project 
teams, this lack of awareness of their peers' methodologies and paradigms was noteworthy and disconcerting. Interestingly, it was more often the social scientists who discussed the need to cross such interdisciplinary boundaries and to muster the humility to do so; never did a biophysical scientist discuss such a need. Miller et al. (2008) explained this duality, "Most interdisciplinary research ends up entitling a single discipline or epistemology, incorporating others in a support or service role-we can refer to this as 'epistemological sovereignty"' (p. 46). In the SP, in particular, the faculty members interviewed described seeing epistemological sovereignty of the hard sciences over the soft sciences. In this way, the social scientists in the SP play an interesting subservient role to the biophysical scientists, providing a service not only to what is considered the dominant perspective in the work, but also to provide a bridge to create such interdisciplinary efforts in the first place. Those working with the SP may consider providing opportunities for the faculty members to understand the individual disciplinary contributions to the larger goals of the project and to provide structured time and opportunities for understanding language, methodology, and alternate paradigmatic assumptions. Particularly important are such efforts for interdisciplinary endeavors related to sustainability, as these have typically been dominated by the natural sciences, despite the vital need for social science and critical perspectives that the natural sciences traditionally do not offer (Clark et al. 1999; Jerneck et al. 2011; Quinlan and Scogings 2004; Stock and Burton 2011; van Kerkhoff 2005).

Related to the chasm between the hard and soft sciences was the theme of the status 
and hierarchy inherent in these classifications. Part of this status and power is inherent in a discipline's openness to outside influences. Becher and Trowler (2001) discussed this issue in respect to the applied fields and to the soft-applied fields, in particular. They remarked that these soft disciplines are "seen internally as politically weak and externally as lacking in good intellectual standing...this loss of self-confidence has rendered the social sciences especially vulnerable to attack from unsympathetic external forces" (p. 192). As a result, they stated that many of these soft disciplines often undergo efforts to make themselves lean more toward a hard paradigmatic stance. They commented, "'Academic drift' continues to exercise a strong attraction for those who are too low in the pecking order for their own comfort" (p. 193). In this way, economics played an interesting role in the SP, which also showed up in their own placement in the Guba (1990) framework. Becher and Trowler echoed the interesting role that economics plays in the larger classification of the disciplines, discussing it as a discipline that straddles both worlds of hard and soft, as well as pure and applied. In turn, economists were prevalent among the many funded subprojects under the SP. Perhaps it is these "straddling" disciplines that will ultimately find the most success in transitioning smoothly to interdisciplinary endeavors.

These issues of status and power may also play important roles in interdisciplinary endeavors as a whole. Interdisciplinarity is, by its nature, a risky undertaking (Amey and Brown 2004), as it challenges prevailing organizational, social, and political structures and traditions. Given the highly tradition-oriented nature of academia, in particular, interdisciplinary research collaborations take on an interesting new light. Becher and 
Trowler (2001) discussed the resistance to change that is often present in many disciplines. Many explanations can be offered for this conservatism but power certainly stands as a salient one, given the dominance of particular paradigms and the existing social structures to support their continuance (Becher and Trowler 2001). At the same time, Becher and Trowler posited something else at work in terms of those disciplines that might be relatively more open to paradigmatic changes: "Those who have as yet made no major intellectual commitments have little to lose by investing in potentially high-risk, high-profit commodities; those who already have a substantial blue chip portfolio tend to see the emergence of rival markets as a threat rather than a promise" (p. 99). In other words, perhaps those disciplines that already operate at a lower status-perhaps due to their lack of paradigmatic consensus - are those most open to these types of revolutionary changes that true interdisciplinary work entails, whereas those in the hard science or biophysical fields are reticent to give up their foothold on the stability of status they have achieved.

An additional paradox then exists, however. To gain reputation and prestige and, consequently, more power within the disciplinary hierarchy, one must make the groundbreaking new discoveries that prompt such attention (Becher and Trowler 2001). The work of interdisciplinarity, which operates at these interstices among disciplines and paradigms, has the promise to afford such ground-breaking discoveries. It is, perhaps, the strength of the organizational boundaries and the social communities that guard them that will ultimately influence one's ability to cross them. In this way, it is perhaps not surprising that those in the soft disciplines were more likely to feel at once tentative but 
also more open to the idea of paradigm- crossing interdisciplinarity, whereas those in the hard disciplines seemed the most reticent to do so. It is only perhaps with this continued openness to different approaches, methods, and paradigms that efforts such as the SP can move beyond a mere collaboration among disciplines to a more transformative notion of transdisciplinarity in their sustainability work (Stock and Burton 2011).

The implications from this study are multifaceted. For those who seek to foster interdisciplinary research collaboration that crosses paradigmatic boundaries, it is important to create space, time, and a corresponding reward system to encourage this boundary crossing in both universities and research institutions. Unless hired to do so, many researchers will be attempting to do this kind of work "on top of" the other work in which they are currently engaged (Pohl 2005). If this work is not valued in terms of promotion and tenure policies, as well as financially incentivized, researchers may not be able to invest the required time to learn others' languages, methodologies, and ways of knowing to make such endeavors successful. Only through the extensive time required to do so will researchers be able to understand, and, therefore, respect their colleagues' contributions to interdisciplinary collaborations and to perhaps move toward a more transdisciplinary approach.

In regard to future research, much more has yet to be explored in relation to interdisciplinary collaboration. Ironically, a criticism of the existing literature related to interdisciplinarity also centers around this soft-hard divide. MacMynowski (2007) 
explained, "Simply stated, most of the social scientists are discussing the means of interdisciplinarity without an end in sight whereas the biophysical scientists are ardently promoting an end without deeper consideration of the means involved" (p. 23). Therefore, crossing the disciplinary divide on interdisciplinarity is also warranted. How can the literature in the biophysical sciences inform the literature in the social sciences and vice versa? Moreover, understanding that it is both the process and the means to create this process is fundamental to future research.

Future research can build off of the current study by examining how institutional context influences these kinds of endeavors, including the size of the institution, ranking of the institution, and institutional type. Further, policies related to promotion and tenure could also be examined from institutional contexts that have explicit guidelines to support this work, as opposed to those without such policies in place. Other studies could examine how different combinations of disciplines, demographic characteristics of the faculty participating in them-such as gender and race-and background characteristics mediate interdisciplinary outcomes and experiences. Finally, more research must be conducted on socialization to interdisciplinarity, including related constructs such as attitudinal correlates like tolerance to ambiguity and cognitive development, as discussed by scholars such as Repko (2008) and Klein (1990). MacMynowski (2007) aptly summarized:

If the goal is to expand interdisciplinary research between social and biophysical sciences, it is not enough to merely identify the philosophical, structural, and conceptual differences that surface at their confluence. It is 
essential to take the next step and ask why the difference is there and what purposes are served. In this way, we can go forward with greater transparency, mutual respect, and success in integrating diverse forms of knowledge. (p. 21). 


\section{ACKNOWLEDGMENTS}

Supported by National Science Foundation award EPS-0904155 to Maine EPSCoR

Sustainability Solutions Initiative at the University of Maine. 


\section{REFERENCES}

Amey MJ, Brown DF (2004) Breaking out of the box: interdisciplinary collaboration and faculty work. Information Age, Greenwich Aram JD (2004) Concepts of interdisciplinarity: configurations of knowledge and action. Hum Relat 57:379-412

Becher T (1981) Towards a definition of disciplinary cultures. Stud High Educ 6:109-122

Becher T (1989) Academic tribes and territories: intellectual enquiry and the culture of disciplines. Society for Research into Higher Education (SRHE) and Open University Press, Bristol

Becher T, Trowler PR (2001) Academic tribes and territories. Open University Press, Philadelphia

Berliner DC (2002) Comment: educational research: the hardest science of all. Educ Res $31: 18-20$

Biglan A (1973a) The characteristics of subject matter in different academic areas. J Appl Psychol 57:195-203

Biglan A (1973b) Relationships between subject matter characteristics and the structure and output of university departments. J Appl Psychol 57:204-213

Bogdan RC, Biklen SK (2003) Qualitative research for education: an introduction to theories and methods. Allyn and Bacon, Boston

Borrego M, Newswander LK (2010) Definitions of interdisciplinary research: toward graduate-level interdisciplinary learning out-comes. Rev High Educ 34:60-84

Clark BR (1987) The academic life: small worlds, different worlds. Carnegie Foundation for the Advancement of Teaching, Princeton

Clark WC, Kates RW; Members of the Board on Sustainable Development of the National Research Council (1999) Our common journey: a transition toward sustainability. National Academy Press, Washington, DC

Creamer EG (2003) Exploring the link between inquiry paradigm and the process of collaboration. Rev High Educ 26:447-465

Derry SJ, Schunn CD (2005) Interdisciplinarity: a beautiful but dangerous beast. In: Derry SJ, Schunn CD, Gernsbacher MA (eds) Interdisciplinary collaboration: an emerging cognitive science. Lawrence Erlbaum Associates, Mahwah, pp xiii-xx

Dressel PL, Marcus D (1982) On teaching and learning in college. Jossey-Bass, San Francisco

Frost SH, Jean PM (2003) Bridging the disciplines: interdisciplinary discourse and faculty scholarship. J High Educ 74:119-149

Glaser BG (1978) Theoretical sensitivity: advances in the methodology of grounded theory. Sociology Press, Mill Valley

Golde CM, Gallagher HA (1999) The challenges of conducting interdisciplinary research in traditional doctoral programs. Ecosystems 2:281-285

Guba EG (1990) The alternative paradigm dialog. In: Guba EG (ed) The paradigm dialog. Sage, Newbury Park, pp 17-27

Guba EG, Lincoln YS (1994) Competing paradigms in qualitative research. In: Denzin NK, Lincoln YS (eds) Handbook of qualitative research. Sage, Thousand Oaks, pp 105117 
Hedges LV (1987) How hard is hard science, how soft is soft science? The empirical cumulativeness of research. Am Psychol 42:443- 455

Holley K (2009) Understanding interdisciplinary challenges and opportunities in higher education. Jossey-Bass, San Francisco

Jerneck A, Olsson L, Ness B, Anderberg S, Baier M, Clark E, Hickler T, Hornborg A, Kronsell A, Lövbrand E, Persson J (2011) Structuring sustainability science. Sustain Sci 6:6982

Kates RW, Clark WC, Corell R, Hall JM, Jaeger CC, Lowe I, McCarthy JJ, Schellnhuber HJ, Bolin B, Dickson NM, Faucheux S, Gallopin GC, Grübler A, Huntley B, Jä ger J, Jodha NS, Kasperson RE, Mabogunje A, Matson P, Mooney H, Moore B 3rd, O'Riordan T, Svedin U (2000) Sustainability science. Harvard University Belfer Center for Science and International Affairs, Cambridge

Klein JT (1990) Interdisciplinarity: history, theory, and practice. Wayne State University, Detroit

Klein JT (2005) Interdisciplinary teamwork: the dynamics of collaboration and integration. In: Derry SJ, Schunn CD, Gernsbacher MA (eds) Interdisciplinary collaboration: an emerging cognitive science. Lawrence Erlbaum Associates, Mahwah, pp xiii-xx

Klein JT (2010) Creating interdisciplinary campus cultures: a model for strength and sustainability. Jossey-Bass, San Francisco

Kuhn TS (1962) The structure of scientific revolutions. University of Chicago Press, Chicago

Lattuca LR (2001) Creating interdisciplinarity: interdisciplinary research and teaching among college and university faculty. Vanderbilt University Press, Nashville

MacMynowski DP (2007) Pausing at the brink of interdisciplinarity: power and knowledge at the meeting of social and biophysical science. Ecol Soc 12:20-33

Maxwell JA (1996) Qualitative research design: an interactive approach. Sage, Thousand Oaks

Miller TR, Baird TD, Littlefield CM, Kofinas G, Chapin FS 3rd, Redman CL (2008)

Epistemological pluralism: reorganizing interdisciplinary research. Ecol Soc 13:46-62

National Science Foundation (NSF) (2007) IGERT National Recruitment Program

National Science Foundation (NSF) (2009) Interdisciplinary research Pallas AM (2001)

Preparing education doctoral students for epistemological diversity. Educ Res 30:611

Pohl C (2005) Transdisciplinary collaboration in environmental research. Futures 37:11591178

Quinlan T, Scogings P (2004) Why bio-physical and social scientists can speak the same language when addressing sustainable development. Environ Sci Policy 7:537-546

Repko AF (2008) Interdisciplinary research: process and theory. Sage, Thousand Oaks

Rossini FA, Porter AL, Kelly P, Chubin DE (1979) Frameworks and factors affecting integration within technology assessments. In: Barth R, Steck R (eds) Interdisciplinary research groups: their management and organization. Interstudy, Seattle, pp 161-196

Schwandt TR (1990) Paths to inquiry in the social disciplines: scientific, constructivist, and critical theory methodologies. In: Guba EG (ed) The paradigm dialog. Sage, 
Newbury Park, pp 258-276

Stock P, Burton RJF (2011) Defining terms for integrated (multi- inter-trans-disciplinary) sustainability research. Sustainability 3:1090-1113

Storer NW (1967) The hard sciences and the soft: some sociological observations. Bull Med Libr Assoc 55:75-84

Strauss AC, Corbin JM (1998) Basics of qualitative research: techniques and procedures for developing grounded theory. Sage, Thousand Oaks

The National Academies (2004) Facilitating interdisciplinary research. The National Academies Press, Washington, DC

Toma JD (1997) Alternative inquiry paradigms, faculty cultures, and the definition of academic lives. J Higher Educ 68:679-705

Van Kerkhoff L (2005) Integrated research: concepts of connection in environmental science and policy. Environ Sci Policy 8:452-463 